06,04

\title{
Температурные свойства диэлектрической проницаемости и оптические свойства многослойной структуры вариозонного титаната бария-стронция
}

\author{
(C) Л.В. Григорьев ${ }^{1,2}$, А.А. Семенов ${ }^{2}$, А.А. Иванов ${ }^{2}$, Е.А. Седых ${ }^{1}$ \\ ${ }^{1}$ Университет ИТМО, \\ Санкт-Петербург, Россия \\ ${ }^{2}$ Санкт-Петербургский государственный электротехнический университет, \\ Санкт-Петербург, Россия \\ E-mail: Ivgrigoryev@itmo.ru
}

Поступила в Редакцию 10 августа 2020 г.

В окончательной редакции 10 августа 2020 г.

Принята к публикации 13 сентября 2020 г.

\begin{abstract}
Представлены результаты исследования оптических свойств и температурной зависимости диэлектрической проницаемости многослойной структуры вариозонного сегнетоэлектрика титаната бария-стронция, нанесенного на монокристаллическую подложку из лейкосапфира. Приведены результаты математического моделирования и экспериментального исследования коэффициента пропускания и отражения структуры в спектральной полосе 300-1100 nm при малых углах падения света на поверхность. Представлены результаты экспериментального исследования температурной зависимости диэлектрической проницаемости в диапазоне температур 300-400 K.
\end{abstract}

Ключевые слова: сегнетоэлектрик, титанат бария-стронция, тонкие пленки сегнетоэлектриков, магнетронное распыление, радиофотоника.

DOI: 10.21883/FTT.2021.01.50404.168

\section{1. Введение}

Переход на радиофотонные методы генерации и управление излучением в СВЧ и $\mathrm{THz}$ полосе позволяет создать адаптивные фазированные антенные решетки с электронным или оптическим сканированием луча в пространстве [1]. Для успешного решения этой задачи необходимо создание оптически и электрические управляемых микроэлектронных структур фазовращателей CВЧ и $\mathrm{THz}$ диапазона на основе новых сегнетоэлектрических материалов и структур [2]. Такие фазовращатели могут быть созданы на базе сегнетоэлектрических материалов (сегнетоэластиков) или сегнетоэлектрических структур, обладающих размытым фазовым переходом в широком температурном диапазоне и имеющих малые потери в диапазоне СВЧ и $\mathrm{THz}$ [3]. В этой связи становится весьма актуальной задача по созданию многослойных структур на основе вариозонного титаната бария-стронция, так как он имеет доказано малые потери в СВЧ диапазоне.

В работе представлены результаты численного моделирования и экспериментального исследования оптических свойств и поведения температурной зависимости диэлектрической проницаемости многослойной структуры вариозонного титаната бария стронция.

\section{2. Приготовление образцов}

В качестве подложки для формирования слоистых структур в настоящей работе использовался лейкосап- фир $\mathrm{Al}_{2} \mathrm{O}_{3}$ ориентации [1012] толщиной $0.5 \mathrm{~mm}$. Исследуемые образцы представляли собой монокристаллическую подложку из лейкосапфира с нанесенной на его поверхность многослойной тонкопленочной структурой вариозонного сегнетоэлектрика титаната бариястронция $\mathrm{Ba}_{x} \mathrm{Sr}_{1-x} \mathrm{TiO}_{3}$ (BST). Для формирования пленок и слоистых структур BST в работе использовалась технология ВЧ реактивного магнетронного распыления керамических мишеней [4,5]. Технологическая установка была оснащена тремя магнетронными узлами. В качестве мишеней применялась спеченная керамика твердого раствора $\mathrm{Ba}_{x} \mathrm{Sr}_{1-x} \mathrm{TiO}_{3}$ требуемого стехиометрического состава. Индекса $x$ принимал значения $0.2,0.4$ и 0.7 для отдельных слоев структуры. Процесс осаждения пленок проводился в среде кислорода, при давлении 10-20 Ра; расстояние пленка-подложка устанавливалось в пределах 20-30 mm; удельная мощность ВЧ-разряда составляла 6-10 W/cm ${ }^{2}$; температура подложек лежала в диапазоне $600-700^{\circ} \mathrm{C}$; скорость роста пленок составляла $1.5-2 \mathrm{~nm} / \mathrm{min}(\sim 100 \mathrm{~nm} / \mathrm{h})$ процесс формирования многослойной структуры заключался в последовательном, поочередном распылении мишеней с требуемой стехиометрией. В процессе послеростовой обработки сформированной слоистой структуры проводился отжиг в атмосфере кислорода при температуре $900^{\circ} \mathrm{C}$ в течении двух $\mathrm{h}$ для пассивации кислородных вакансий в $\mathrm{Ba}_{x} \mathrm{Sr}_{1-x} \mathrm{TiO}_{3}$, образующихся в процессе ионноплазменного распыления керамических мишеней. В каждом слое BST варьировалось процентное содержание 

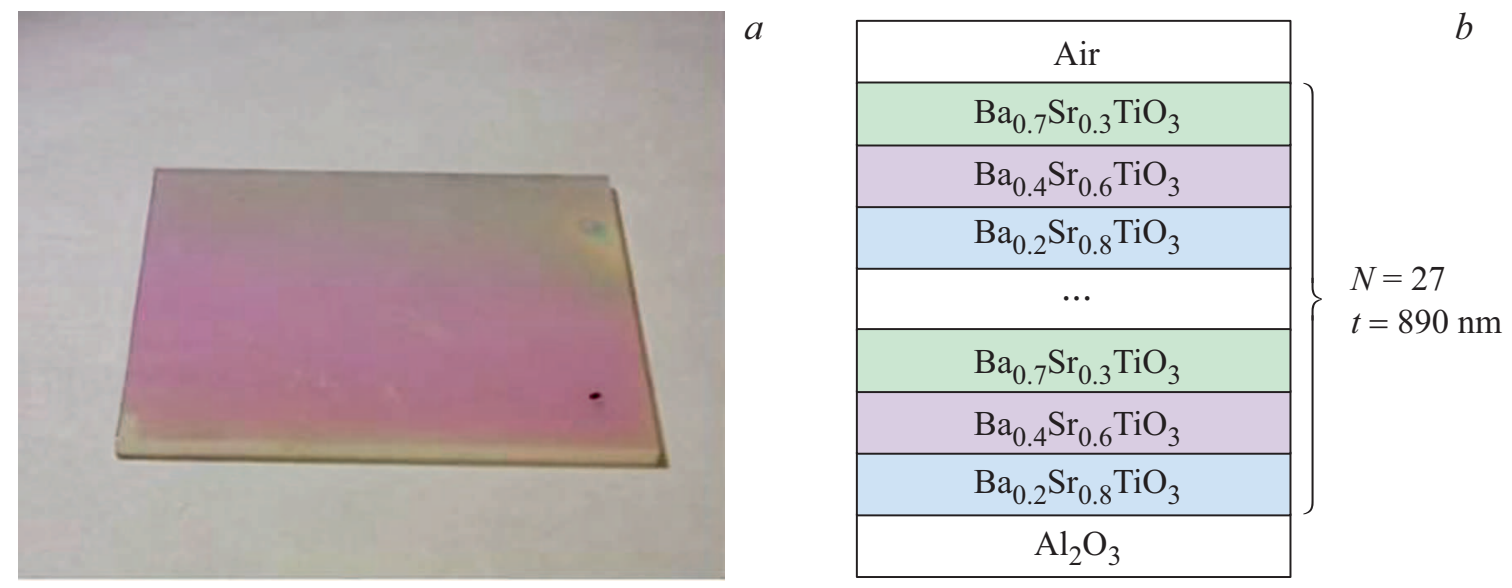

Рис. 1. $a-$ внешний вид исследуемой структуры. $b-$ поперечное сечение структуры.

бария/стронция. Структура состояла из 27 слоев BST, в которых последовательно изменялся $x: x=0.2,0.4$ и 0.7 . Общая толщина структуры составляла $860 \mathrm{~nm}$. Внешний вид структуры и его послойной вид представлен на рис. 1 ( $a-$ фото внешнего вида; $b-$ поперечное сечение).

\section{3. Исследование оптических свойств многослойной структуры}

Спектральные зависимости коэффициента пропускания представлены на рис. 2 и спектральная зависимость коэффициента пропускания на рис. 3 (кривая $1-$ результат численного моделирования, кривая 2 - экспериментальный результат). Было проведено численное моделирование коэффициента пропускания в спектральной полосе $500-1100 \mathrm{~nm}$. Для расчета использовалась модель многослойного диэлектрика, оптические свой-

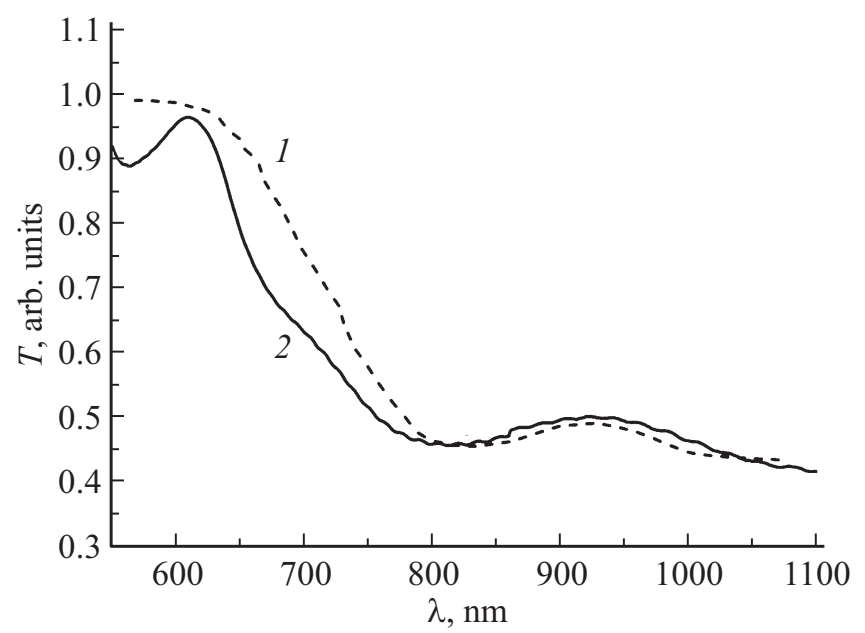

Рис. 2. Спектральная зависимость коэффициента пропускания исследуемой структуры: 1 - численное моделирование, 2 - эксперимент. ства диэлектрических слоев которого были взяты для находящегося при комнатной температуре $\mathrm{Ba}_{x} \mathrm{Sr}_{1-x} \mathrm{TiO}_{3}$,

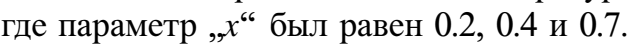

Численное моделирование спектральной зависимости коэффициента пропускания $T(\lambda)$ и коэффициента $R(\lambda)$ отражения рассчитывалась по методике распространения света в многослойных диэлектрических системах, приведенной в работе [6].

Результат численного расчета зависимости $T(\lambda)$ в спектральной полосе $500-1100 \mathrm{~nm}$ представляет собой сложную кривую, состоящую из нескольких областей: области монотонного спада и области локального максимума. В диапазоне длин волн $500-630 \mathrm{~nm}$ величина коэффициента пропускания многослойной структуры лежит в диапазоне значений $0.98-0.96$. Начиная с длины волны $635 \mathrm{~nm}$ наблюдается резкое уменьшение коэффициента пропускания, которое на длине волны 790 нам достигает своего минимального значения, равного 0.45 . В спектральной полосе $795-850 \mathrm{~nm}$ величина коэффициента пропускания практически не изменяется и составляет $0.45-0.46$. В спектральной полосе $858-1010 \mathrm{~nm}$ наблюдается локальное увеличение $T(\lambda)$, т. е. локальный максимум коэффициента пропускания, величина которого равна 0.51 . После $1010 \mathrm{~nm}$ имеет место монотонный спад коэффициента пропускания до уровня 0.4.

Результат численного расчета зависимости $R(\lambda)$ в спектральной полосе $500-1100 \mathrm{~nm}$ представляет собой монотонную кривую (рис. 3, кривая 1), состоящую из двух квазилинейных областей. В спектральной полосе $550-750 \mathrm{~nm}$ наблюдается рост коэффициента отражения от 0.023 до 0.18 и далее, в области от $750 \mathrm{~nm}$ до $1100 \mathrm{~nm}$ наблюдается монотонное увеличение $R(\lambda)$ до величины 0.21 .

Экспериментальное исследование спектральных зависимостей коэффициентов пропускания и отражения в диапазоне длин волн $0.5-1.1 \mu \mathrm{m}$ проводилось на спектрофотометре Perkin Elmer при комнатной температуре. Экспериментальная зависимость $T(\lambda)$ представлена на рис. 2 (кривая 2). Вид $T(\lambda)$ для исследуемой много- 


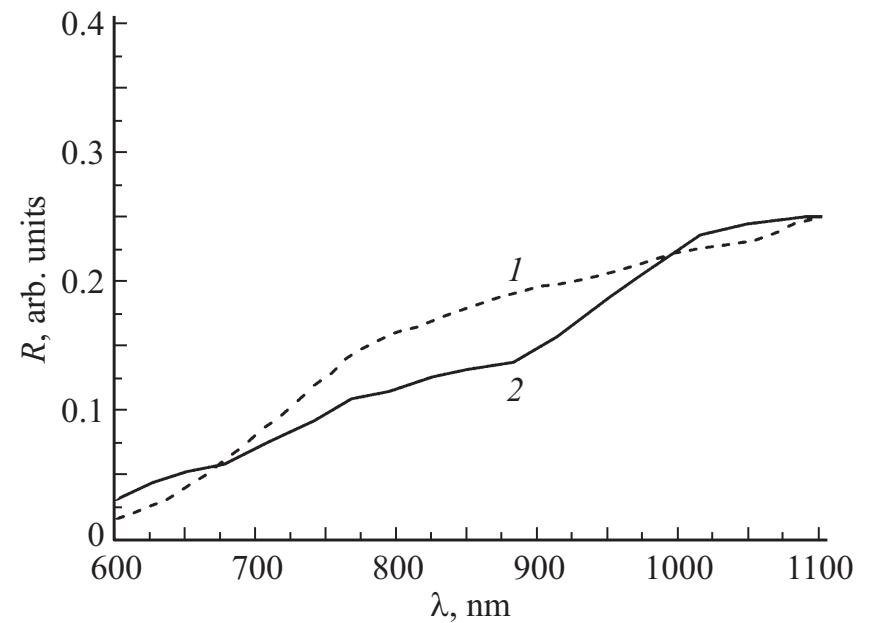

Рис. 3. Спектральная зависимость коэффициента отражения исследуемой структуры: 1 - численное моделирование, 2 - эксперимент.

слойной сегнетоэлектрической структуры незначительно отличается от вида $T(\lambda)$, полученного численным моделированием и рассмотренного нами выше. Так в спектральной полосе 560-675 nm на кривой спектральной зависимости коэффициента пропускания наблюдается пик, максимум которого находится на длине волны $630 \mathrm{~nm}$ и максимальное значение коэффициента пропускания многослойной диэлектрической структуры равно 0.96. Далее, в спектральной полосе 680-790 nm наблюдается спад величины коэффициента пропускания по сублинейному закону до величины 0.45 . В диапазоне длин волн от 800 до $850 \mathrm{~nm}$ на кривой коэффициента пропускания наблюдаются слабые осцилляции, которые могут быть связаны с наблюдаемым эффектом интерференции в многослойной структуре. В этом спектральном промежутке коэффициент пропускания сохраняет неизменное значение. В спектральной полосе 850-1020 nm на кривой $T(\lambda)$ исследуемой структуры наблюдается локальный максимум коэффициента пропускания. Положение максимума, величина максимального значения $T(\lambda)$ и энергетическая полуширина локального максимума практически совпадает с положением, энергетической полушириной и величиной максимума коэффициента пропускания, полученных с помощью численного моделирования.

Экспериментальная зависимость $R(\lambda)$ в спектральной полосе 500-1100 nm представляет собой сложную кривую (рис. 3, кривая 2), состоящую из четырех квазилинейных участков. В спектральной полосе $550-780 \mathrm{~nm}$ коэффициент отражения увеличивается от 0.026 до 0.1 . В спектральной полосе 790-880 $\mathrm{nm} R(\lambda)$ незначительно увеличивается в диапазоне значений от 0.11 до 0.13 со скоростью роста в 1.8 раза меньше, чем в рассмотренной ранее спектральной полосе. В спектральной области 890-1025 nm наблюдается резкое возрастание величины коэффициента отражения от 0.13 до 0.23 , причем ско- рость увеличения $R(\lambda)$ становится больше в 2.3 раза, чем в предыдущей спектральной области. В диапазоне длин волн 1025-1100 nm наблюдается область с наименьшей скоростью увеличения (роста) $R(\lambda)$, т. е практически в этой спектральной полосе на графике $R(\lambda)$ наблюдается область „плато“ и коэффициент отражения изменяется на 0.02 от величины 0.23 до 0.25 . Влияния развитого интерфейса межфазных границ (между слоями с различным соотношением бария к стронцию) на оптическое рассеяние в полосе 550-1100 nm нами не было экспериментально обнаружено.

\section{4. Исследование температурной зависимости диэлектрической проницаемости многослойной структуры}

Предварительно было проведено численное моделирование температурной зависимости диэлектрической проницаемости многослойной структуры титаната бариястронция. Многослойная структура рассматривалась как система из последовательно соединенных конденсаторов, у которых изменялось значение диэлектрической проницаемости [7-9]. Вид экспериментально измеренных температурных зависимостей $\varepsilon(T)$ для образцов $\mathrm{Ba}_{x} \mathrm{Sr}_{1-x} \mathrm{TiO}_{3}$ с вариацией отношения бария к стронцию приведен на рис. $4\left(\mathrm{Ba}_{x} \mathrm{Sr}_{1-x} \mathrm{TiO}_{3}\right.$ для каждого случая: $x=0.2,0.4$ и 0.7). Краевыми эффектами на границе образца пренебрегали. Результат численного моделирования приведен на рис. 5 (график 2). График численного моделирования $\varepsilon(T)$ представляет собой сложную кривую, состоящую из нескольких функциональных зависимостей: в диапазоне температур $20-35^{\circ} \mathrm{C}$ наблюдается линейный рост температурной зависимости диэлектрической проницаемости. От 38 до $45^{\circ} \mathrm{C}$ наблюдается экспоненциальный рост $\varepsilon(T)$ и далее в диапазоне от 48 до $97^{\circ} \mathrm{C}$ кривая $\varepsilon(T)$ имеет вид многогорбой

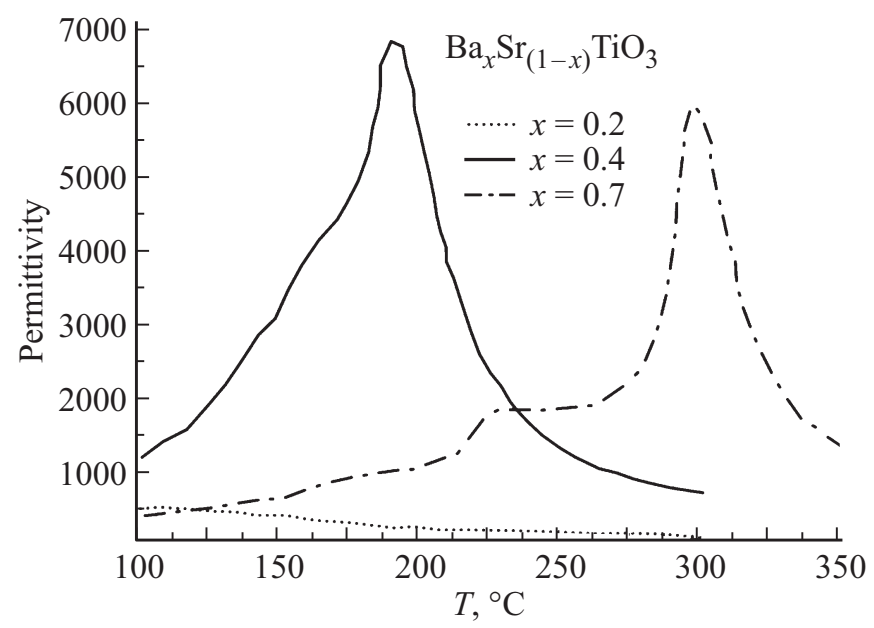

Рис. 4. Температурная зависимость диэлектрической проницаемости $\mathrm{Ba}_{x} \mathrm{Sr}_{1-x} \mathrm{TiO}_{3}$ с различным соотношением $\mathrm{Ba} / \mathrm{Sr}$. 


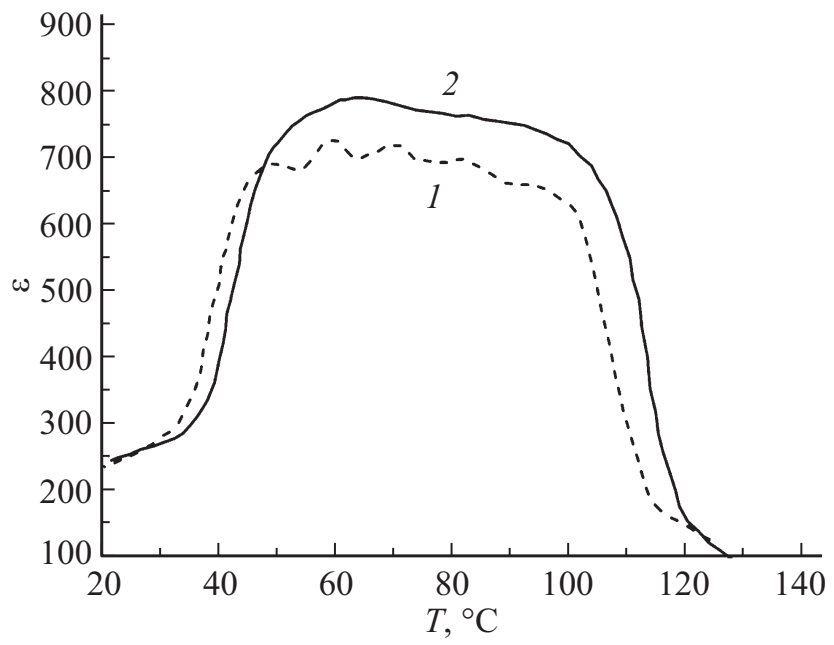

Рис. 5. Температурная зависимость диэлектрической проницаемости исследуемой структуры: 1 - численное моделирование, 2 - эксперимент.

кривой с незначительными вариациями „пиков“ $\varepsilon(T)$ относительно максимального значения диэлектрической проницаемости. Наличие на графике нескольких локальных максимумов („пиков“) может быть объяснено парциальным вкладом зависимостей $\varepsilon(T)$ от слоев $\mathrm{Ba}_{x} \mathrm{Sr}_{1-x} \mathrm{TiO}_{3}$ с различными значениями параметра $x$. После температуры $100^{\circ} \mathrm{C}$ на графике $\varepsilon(T)$ наблюдается экспоненциальное уменьшение $\varepsilon$ и в диапазоне от 115 и до $127^{\circ} \mathrm{C}$ наблюдается линейное уменьшение зависимости диэлектрической проницаемости. Такое поведение $\varepsilon(T)$ может быть обусловлено, тем что температура Кюри $\mathrm{Ba}_{x} \mathrm{Sr}_{1-x} \mathrm{TiO}_{3}$ не превышает $120^{\circ} \mathrm{C}$, поэтому все слои титаната бария-стронция находятся в параэлектрической фазе и их суммарных парциальный вклад в общую зависимость $\varepsilon(T)$ приводит к сублинейной зависимости.

Экспериментальное исследование температурной зависимости диэлектрической проницаемости многослойной структуры из вариозонного $\mathrm{Ba}_{x} \mathrm{Sr}_{1-x} \mathrm{TiO}_{3}$ было проведено на RLC-метре HIOKI 3335 в РЧ-экранированной ячейке, в температурном диапазоне $20-140^{\circ} \mathrm{C}$, без освещения образца. Частота измерительного сигнала составляла $1 \mathrm{~Hz}$. Экспериментальная кривая зависимости диэлектрической проницаемости от температуры приведена на рис. 5 (график № 1). Зависимость $\varepsilon(T)$ также представляет собой сложную кривую, состоящую из нескольких функциональных зависимостей: в диапазоне температур $20-37^{\circ} \mathrm{C}$ наблюдается линейный рост температурной зависимости диэлектрической проницаемости, при котором наблюдается незначительный рост $\varepsilon: 248$ до 290. В температурном диапазоне от 40 до $50^{\circ} \mathrm{C}$ наблюдается экспоненциальный рост $\varepsilon(T)$ с существенным увеличением $\varepsilon$ : диэлектрическая проницаемость многослойной структуры возрастает от 300 до 750. Далее в диапазоне температур от 55 до $65^{\circ} \mathrm{C}$ наблюдается плавный выход на максимум, где при температуре $65^{\circ} \mathrm{C} \varepsilon$ достигает величины 788. В температурном диапазоне $66-100^{\circ} \mathrm{C}$ имеет место монотонное уменьшение величины диэлектрической проницаемости от значения 788 до 701. Дальнейшее увеличение температуры приводит к экспоненциальному спаду на графике $\varepsilon(T)$ и в диапазоне температур $103-120^{\circ} \mathrm{C}$ величина $\varepsilon$ изменяется от 700 до 154. В отличие от графика $\varepsilon(T)$, полученного численным моделированием, на экспериментальной зависимости $\varepsilon(T)$ в температурном диапазоне $66-100^{\circ} \mathrm{C}$ отсутствуют ярко выраженные локальные пики.

\section{5. Заключение}

Методом ионно-плазменного распыления керамических мишеней на поверхности полированного лейкосапфира удалось сформировать многослойную структуру из сегнетоэлектрика $\mathrm{Ba}_{x} \mathrm{Sr}_{1-x} \mathrm{TiO}_{3}$, где $x=0.2,0.4$ и 0.7 .

Проведенное численное моделирование спектральных зависимостей коэффициентов пропускания и отражение многослойной структуры BST в полосе 550-1100 nm показало хорошее совпадение с экспериментальными результатами. Этот факт позволяет утверждать о применимости использованной математической модели для прогнозирования оптических свойств многослойных сегнетоэлектрических структур в которых варьируется от слоя к слою соотношение $\mathrm{Ba} / \mathrm{Sr}$.

Величина коэффициента пропускания не ниже 0.45 в спектральной полосе $850-1100 \mathrm{~nm}$ и величина коэффициента отражения, в той же спектральной полосе, лежащая в диапазоне значений от 0.15 до 0.25 , позволяют использовать исследуемую структуры в приложениях радиофотоники, волоконной и интегральной оптики с лазерной накачкой от полупроводниковых гетеролазеров или DPSS-лазеров YAG: Nd.

Температурная зависимость диэлектрической проницаемости исследуемой структуры показала наличие размытого фазового перехода, свойственного сегнетоэлектрикам-сегнетоэластикам. Сравнение результатов численного моделирования поведения $\varepsilon(T)$, основанных на модели конденсатора с многослойным диэлектриком из различных сегнетоэлектрических слоев показало хорошее совпадение с результатами экспериментальных измерений температурной зависимости диэлектрической проницаемости в исследуемой многослойной структуре. Наличие широкой температурной области: $45-110^{\circ} \mathrm{C}$, где значение $\varepsilon(T)$ изменяется в пределах 7\% от максимума позволяет использовать сформированную структуру для создания фазовращаетелей.

\section{Конфликт интересов}

Авторы заявляют,что у них отсутствует конфликт интересов. 


\section{Список литературы}

[1] Г. Вендик, М.Д. Парнес. Антенны с электрическим сканированием. Введение в теорию. Сайнс-пресс, М. (2002).

[2] A.A. Nikitin, A.B. Ustinov, V.V. Vitko, A.A. Semenov, P.Y. Belyavskiy, I.G. Mironenko, A.A. Stashkevich, B.A. Kalinikos, E. Lähderanta. J. Mater. Sci. 51, 17, 7803 (2016).

[3] В.М. Балашов, И.Г. Мироненко, А.И. Фирсенков, А.А. Иванов, Д.В. Велькин, А.А. Семенов. Вопросы радиоэлектроники 9, 67 (2019).

[4] Б.С. Данилин, В.К. Сырчин Магнетронное распылительные системы. Радио и связь, М. (1982).

[5] Л. Холлэнд Нанесение тонких пленок в вакууме. Госэнергоиздат, М. (1963).

[6] Э.С. Путилин, Оптические покрытия. ИТМО, СПб (2005). $199 \mathrm{c}$.

[7] А.А. Семенов, А.И. Дедык, И.Л. Мыльников, О.В. Пахомов. ФТТ 57, 3, 523 (2015).

[8] A.A. Semenov, A.I. Dedyk, Y.V. Pavlova, P.Y. Beliavskiy, I.L. Mylnikov. Ferroelectrics. 447, 1, 117 (2013).

[9] O.G. Vendik, S.P. Zubko, M.A. Nikol'ski. J. Appl. Phys. 92, 12, 7448 (2002).

[10] O.G. Vendik, S.P. Zubko. J. Appl. Phys. 82, 9, 4475 (1997).

Редактор К.В. Емиев 